\title{
CONCEPTUAL FRAMEWORK ON USE OF GENERATING FUNCTIONS FOR MODELLING THE PAYMENT PROCESS AT CASH REGISTER
}

\author{
Detlef Hartleb ${ }^{1}$, Andreas Ahrens ${ }^{2}$ and Jelena Zascerinska ${ }^{3}$ \\ ${ }^{I}$ Escuela Tecnica Superior de Ingeneria y Sistemas de Telecomunicacion, Universidad Politecnica de Madrid \\ Technical University of Madrid, Crtra de Valenica, $\mathrm{km}$ 7, Madrid, Spain \\ ${ }^{2}$ Hochschule Wismar, University of Technology, Business and Design, Philipp-Müller-Straße 14, 23966 Wismar, \\ Germany \\ ${ }^{3}$ Centre for Education and Innovation Research, Dammes iela 33-102, Riga, Latvia
}

\begin{abstract}
Queuing at the cash register still remains an issue that increases customers' dissatisfaction. Many researchers focused their efforts on preventing a queue through the advancement of the efficiency of technical equipment for the payment processing. Analysis of the payment process has not received a proper attention from the scientific community. The aim of this paper is to present the conceptual framework on the use of generating functions for modelling the payment process at the cash register. The methodological foundation of the work is based on binary customer behavior. The theoretical interconnections between the payment process at the cash register, queuing, bottlenecks, burstiness, gap processes, generating function established by the authors serve as the conceptual framework on the use of generating functions for modelling the payment process at the cash register. The conceptual framework proposed by the authors allows analyzing the payment process as the combination of the processes of buyers' waiting to the cash register described by free time intervals between buyers as well as the payment processing time (also referred as buyers' service time). Generating functions were used for the analysis of the free time intervals between buyers to the cash register as a part of the payment process. Directions of further research are formulated.
\end{abstract}

\section{KEYWORDS}

Payment Process at the Cash Register, Queueing, Bottlenecks, Burstiness, Gap Processes, Generating Function

\section{INTRODUCTION}

Queuing remains an issue in many businesses as queues serve as a reason of customers' dissatisfaction. Queues appear due to a bottleneck emergence. In business systems, bottlenecks limit the flow of customers, services or products, etc. A bottleneck emerges when single business processes within the business system operate at their capacity limit or beyond. In turn, a bottleneck is apparent when a single business process, i.e. the payment process at the cash register, is of bursty nature. Bursts correspond to an enhanced activity level over a short period of time followed by long periods of inactivity. Conventionally, bottlenecks can be measured by indicators or parameters such as the buyers' probability and buyers' concentration (Ahrens et al, 2019).

In order to increase customers' loyalty and attract new customers, companies search for the new ways and measures to optimize their business processes including the payment process at the cash register. The optimization of bursty business processes requires stochastic models with measurable parameters. Often simplifications worked out in the analysis of such models have led to inaccuracies when events occur in a bursty manner. In order to model bursty business systems accurately when optimizing the performance of such systems using e. g. the well-known queuing theory, stochastic processes with measurable parameters have to be found. Such optimizations have been studied successfully when analyzing bit-errors in telecommunication systems (e.g. wireless systems) (Wilhelm, 1976; Wilhelm, 2018) as well as packet arrivals in Ethernet-based data networks (Kessler et al, 2000) or when analyzing the internet traffic (Kresch and Kulkarni, 2011; Zuckermann, Neame and Addie, 2003), where data packets arrive in bursts as well. 
Given the diversity of systems in which burstiness emerges, the modelling of burstiness plays an important role as bottlenecks are still an indicator of queue emergence. For analysing bursty processes, different approaches such as continuous or discrete event simulation $(\mathrm{Li}, 1992)$ have attracted a lot of attention in the research community. Whereas in continuous simulation the system state is changing over the time, discrete event simulation such as agent-based simulation is using systems events which are going to change the system state.

Previous research efforts in investigating the payment process at the cash register with the aim of the increasing customers' satisfaction mostly were devoted to the advancement of the efficiency of technical equipment for the payment processing:

- $\quad$ using RBAC to secure payment process in cloud (Wang, Cao and Zhang, 2020), or

- $\quad$ time efficiency of Point-Of-Sale payment methods (Polasik et al, 2010), etc.

Against this background, analysis of buyers' burstiness using generating functions could be beneficial for the modelling of the payment process at the cash register.

The aim of this paper is to present the conceptual framework on the use of generating functions for modelling the payment process at the cash register. It should be noted that, by a conceptual framework, the unity of concepts that are used for a particular study is meant (Ahrens and Zaščerinska, 2014).

The methodological foundation of the present research is formed by binary customer behaviour, namely "to buy, or not to buy" (Ahrens and Zaščerinska, 2016). Within the present work, by customers, shop visitors and buyers are meant. A shop visitor ("not to buy") is someone who visits the shop, but does not buy anything in this shop, and a buyer ("to buy") is defined as one who has purchased something in the shop (Hartleb et al, 2020 a; Hartleb et al., 2020 b).

The novelty of this paper is revealed by the authors' proposal of the conceptual framework on the use of generating functions applied to the system model, namely free time intervals between buyers waiting to the cash register, for simulating bursty buyers' behaviour aimed at modelling of the payment process at the cash register.

\section{BURSTY PAYMENT PROCESS AT THE CASH REGISTER}

Burstiness in the payment process, as studied in this work, can be addressed when the components have a measurable activity pattern (such as to buy or not to buy). The payment process is a phase in the logical and sequential buying process chain consisting of the customer arrival in the shop, the selection of goods, the payment process as well as the customer or buyer departure (Hartleb, et al., 2020a).

The payment process itself can be divided into the process of buyers' waiting to the cash register described by free time intervals between buyers as well as the payment processing time (also referred as buyers' service time). Figure 1 highlights the two parameters influencing the payment process at the cash register (Hartleb et al., 2020 a; Hartleb et al., 2020 b). As the payment processing time is usually determined by technological limits (scanning of the purchased goods, the paying process itself) and the experience of the sales staff, only the free time intervals between the buyers to the cash register as an indicator of bottlenecks throughout the whole process of buying are studied in this work.

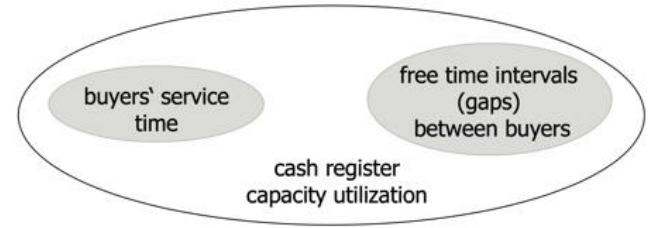

Figure 1. Parameters influencing cash register capacity utilization

The free time intervals between buyers to the cash register, as indicated in Figure 2, as well as the payment processing time intervals at the cash register can be described and modelled by gaps. 


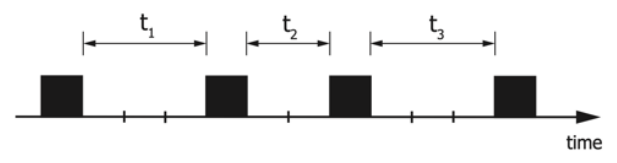

Figure 2. Free time intervals at the cash register

Analysing the gaps between the buyers, i.e. the free time intervals between the buyers to the cash register as shown in Figure 3 allow obtaining such parameters as the buyers' probability and buyers' concentration. Here, stochastic processes with measurable parameters are needed when analysing the free time intervals between the buyers. A process will appear bursty if the probability of short gaps is higher and lower for longer gaps if compared with a process with no burstiness. This results in many short intervals (gaps) of high activity (probability) separated by longer intervals (gaps) of inactivity.

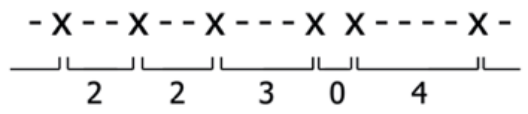

Figure 3. Modelling free-time intervals between the buyers to the cash register (a buyer (represented by "x") within a sequence of non-buying visitors (represented by "-"))

The gaps are characterized by the gap distribution function $u(k)$, defined as the probability that a gap $\mathrm{Y}$ between two buyers is greater than or at least equal to a given number of non-buying visitors $k$, i.e. $u(k)=P(Y \geq k)$.

Intuitively, the buyers' behaviour can be described by a probability of purchase or buyer probability $p_{\mathrm{e}}$ (as a percentage of the visitors who buy something in the shop). However, the buyer probability does not give any indication of how concentrated the buyers are (Ahrens, et al, 2019). In this case, the model has to be extended by at least a second parameter as shown by Wilhelm (Wilhelm 1976; Wilhelm, 2018) and Ahrens (Ahrens, Purvinis and Zaščerinska, 2019) by introducing buyer concentration $(1-\alpha)$. As shown in (Wilhelm 1976; Wilhelm, 2018; Ahrens et al, 2019; Ahrens, Purvinis and Zaščerinska, 2019), a good gap distribution function for bursty as well as non-bursty buyers' behaviour is given by

$$
u(k)=\left[(k+1)^{\alpha}-k^{\alpha}\right] \cdot \mathrm{e}^{-\beta \cdot k} .
$$

depending on the buyer probability $p_{\mathrm{e}}$ and the buyer concentration $(1-\alpha)$. The parameter $\beta$ defined in (1) has to fulfil the following equation

$$
p_{\mathrm{e}} \approx \beta^{\alpha}
$$

as shown by (Wilhelm 1976; Wilhelm, 2018). Practically, relevant buyer concentration is in the range of $0.0<(1-\alpha) \leq 0.5$, whereas the buyer concentration of $(1-\alpha)=0$ describes the situation with non-bursty buyers (also refers to memoryless buyer scenario), where the buyer probability is sufficient to describe the buying process.

Often the performance has been studied under the assumption that the gaps are exponentially distributed. However, under bursty conditions such exponential gap distribution functions become inaccurate (Feldmann, 2000; Kessler et al, 2000; Ahrens et al, 2019; Ahrens, Purvinis, Zaščerinska, 2019).

Thus, for modelling bursty behaviour, the gap distribution function should be different from the exponential one. It should be noted that the statistical properties of the buying process are well approximated by distribution functions with two parameters (Weibull, Wilhelm), whereas distribution functions with only one single parameter (Exponential) provide not such a good result (Ahrens, Purvinis and Zaščerinska, 2018).

Against this background, in this work an alternative approach for modelling bursty as well as non-bursty business processes by using generating functions, which will be used to describe the payment process, is presented. The concept of generating functions is well-developed in the research community. Generating functions have been identified as an extremely beneficial tool when analyzing discrete sequences of infinite length (Zhang, Shen and Waguespack, 2016; Li, 1992). Instead of analyzing a sequence of infinite length, a 
single function can be defined. This single function represents the sequence of infinite length. For example, the sequence $u(k)=(1,1,1,1, \ldots)$ can be represented by the power series

$$
U(t)=\sum_{k=0}^{\infty} u(k) t^{k}=1+t+t^{2}+\cdots .
$$

This power series converges for $|t|<1$ to

$$
U(t)=\sum_{k=0}^{\infty} u(k) t^{k}=\frac{1}{1-t} .
$$

The function $U(t)$ represents the sequence $u(k)=(1,1,1,1, \ldots)$ of infinite length. The elements of the sequence $u(k)$ are the coefficients of the infinite polynomial. Such closed form solutions are known for exponential gap distribution functions. However, it is rather difficult to find closed-form solutions for gap distribution function that are different from the exponential one (Wilhelm 1976; Wilhelm, 2018).

Unfortunately, no generating function for the gap distribution function $u(k)$, defined in (1), can be derived (Wilhelm 1976; Wilhelm, 2018).

\section{GENERATING FUNCTIONS}

Generating functions can be used for describing an infinite sequence of numbers by treating them as the coefficients of a power series (Zhang, Shen and Waguespack, 2016). The concept of generating functions is known in the scientific community and used in this work for modelling the free time intervals (gaps) between the buyers to the cash register. Bursty as well as non-bursty free time intervals between buyers to the cash register are described by the gap distribution function $u(k)$, defined in (1). The generating function associated to (1) is the power series

$$
U(t)=\sum_{k=0}^{\infty} u(k) t^{k} .
$$

As shown in (Hartleb et al, 2020a), the gap-distribution function defined in (1) has the following generating function

$$
U(t)=\frac{1}{\left(1-e^{-\beta} t\right)^{\alpha}} .
$$

This generating function can be used for describing non-bursty (memoryless) as well as bursty free time intervals between the buyers to the cash register.

\section{GROCERY SHOPS IN LITHUANIA}

In order to get practically relevant data regarding the distribution of gaps, the free time intervals between the buyers to the cash register in two different shops (grocery shop and supermarket) in Lithuania are studied. The buyer probability as well as buyer concentration derived from the obtained data allow identifying bottlenecks during the payment process at the cash register in shop sales.

The histograms of the free time intervals at the cash register for the grocery shop as well as the supermarket are given in Figure 4 and Figure 5. Comparing both figures, it turns out that the free times of the cash register are more bursty in the supermarket. Here, either short free time intervals or significantly longer free time intervals are observed. 


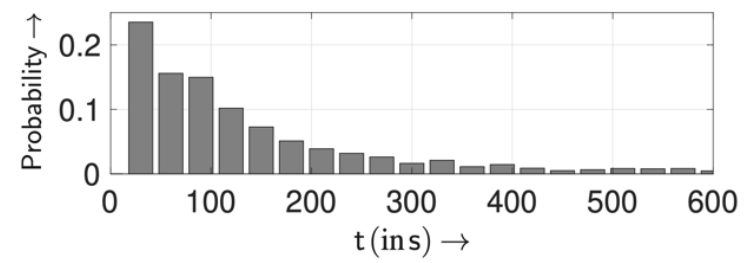

Figure 4. Distribution of free times of cash register (grouped) at grocery shop

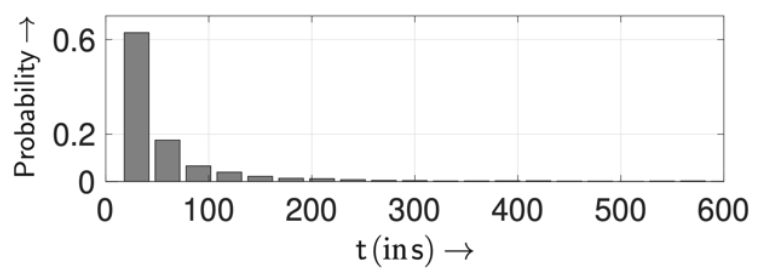

Figure 5. Distribution of free times of cash register (grouped) at the supermarket

\section{CONCLUSIONS}

The theoretical analysis allows establishing the inter-connections between the payment process at the cash register, queuing, bottlenecks, burstiness, gap processes, generating function. The theoretical inter-connections between the payment process at the cash register, queueing, bottlenecks, burstiness, gap processes, generating function established by the authors serve as the conceptual framework on the use of generating functions for modelling the payment process at the cash register. The conceptual framework proposed by the authors allows analysing the payment process as the combination of the processes of buyers' waiting to the cash register described by free time intervals between the buyers as well as the payment processing time (also referred as buyers' service time) as shown in Figure 1.

In this work the concept of generating functions was applied to the field of business processes. Generating functions were used for the analysis of the free time intervals between the buyers to the cash register as a part of the payment process. The proposed generating function offers closed-form solutions. Closed-form solutions are an advantage when using a single function instead of analysing a sequence of infinite length.

The proposed parameters, namely buyer probability and buyer concentration, allow identifying a burstiness level. In turn, a burstiness level serves as an indicator of bottlenecks. A high level of burstiness, expressed by the buyer concentration, increases the possibilities of bottleneck emergence.

For practical verification the payment process was analysed in two shops of different sizes in Lithuania. The obtained results show that in both shops free time intervals at the cash register are quite bursty.

The research has some limitations. A limitation is the theoretical findings based on the analysis of the inter-connections between the payment process at the cash register, queuing, bottlenecks, burstiness, gap processes, generating function. Another limitation is the empirical data collected only in two shops in one country, namely Lithuania.

Future work will be devoted to the design of scenarios based on the inter-play between the processes of buyers' waiting to the cash register as well as the payment processing time (also referred as buyers' service time). Further research will focus on widening the dataset for modelling the payment process at the cash register in order to prevent queuing. Involvement of other business areas, that are different from shops, such as airport, train stations and big hotels would allow comparative studies on a deeper analysis of bustiness for queue prevention as comparative studies bring an added-value research to the scientific community. Comparative studies of different countries are of great research interest, too. 


\section{REFERENCES}

Ahrens, A. et al, 2019. Analysis of a Business Environment using Burstiness Parameter: The Case of a Grocery Shop. In: Edited by Andreas Ahrens, Luis Gomes and César Benavente-Peces, Proceedings of 9th International Joint Conference on Pervasive and Embedded Computing and Communication Systems (PECCS 2019), pp. 49-56. 19-20 September 2019. Vienna, Austria. Printed in Portugal. ISSN: 2184-2817. ISBN: 978-989-758-385-8. Depósito Legal: 460067/19. http://www.peccs.org.

Ahrens, A., Purvinis, O., and Zascerinska, J. 2019. Gap Distributions for Analysing Buyer Behaviour in Agent-based Simulations. In Cesar Benavente-Peces, Andreas Ahrens, Oliver Camp (Editors), Proceedings of the 8th International Conference on Sensor Networks (SENSORNETS 2019), Prague - Czech Republic, Februray 26-27, 2019, pp. 23-29. Printed in Portugal. ISSN: 2184-4380. ISBN: 978-989-758-355-1. Depósito Legal: 451256/19. http://www.sensornets.org.

Ahrens, A., Purvinis, O., Zaščerinska, J. 2018. Gap Structure and Characteristic Properties for Analysing Buyers' Burstiness in E-Business process. In: Edited by Luis Gomes, Andreas Ahrens, César Benavente-Peces and Mohammad S. Obaidat, Proceedings of 8th International Joint Conference on Pervasive and Embedded Computing and Communication Systems (PECCS 2018), pp. 23-34. 29-30 July 2018. Porto, Portugal. Printed in Portugal. ISSN: 2184-2817. ISBN: 978-989-758-322-3. Depósito Legal: 442620/18. http://www.peccs.org.

Ahrens, A., Zaščerinska, J. 2014. A Framework for Selecting Sampe Size in Educational Research on e-Business Application. Proceedings of ICE-B 201411 th International Conference on E-Business, pp. 31-38. Portugal: SciTePress - Science and Technology Publications.

Ahrens, A., Zaščerinska, J. 2016. Gap Processes for Analysing Buyers' Burstiness in E-Business Process. In: Edited by C. Callegari, M. van Sinderen, P. Sarigiannidis, P. Samarati, E. Cabello, P. Lorenz and M. S. Obaidat, Proceedings of the 13th International Joint Conference on e-Business and Telecommunications (ICETE 2016) - Volume 2: ICE-B, pages 78-85. SCITEPRESS Printed in Portugal.

Feldmann, A., 2000. Characteristics of TCP Connection Arrivals. In Park, K. and Willinger, W., editors, Self-similar Network Traffic and Performance Evaluation, chapter 15, pages 367-399. Wiley

Hartleb, D. et al., (2020a). Analysis of Free Time Intervals between Buyers at Cash Register using Generating Functions. In Proceedings of the 10th International Conference on Pervasive and Parallel Computing, Communication and Sensors (PECCS 2020), pages 42-49. ISBN: 978-989-758-477-0.

Hartleb, D. et al., (2020b). Internal and External Factor Analysis in Bottleneck Detection in Shop Sales: The Case of Grocery Shops in Lithuania. In Proceedings of the 10th International Conference on Pervasive and Parallel Computing, Communication and Sensors (PECCS 2020), pages 53-60. ISBN: 978-989-758-477-0.

Kessler, T. et al., 2000. Modelling of connection arrivals in Ethernet-based data networks. In 4rd International Conference on Information, Communications and Signal Processing and 4th IEEE Pacific-Rim Conference on Multimedia (ICICS-PCM), page 3B6.6, Singapore

Kresch, E., Kulkarni, S., 2011. A poisson based bursty model of internet traffic. IEEE 11th International Conference on Computer and Information Technology, pages 255-260

Li, S., 1992. Generating Function Approach for discrete Queueing Analysis with decomposable Arrival and service Markov Chains. In IEEE International Conference on Computer Communications (INFOCOM), pages 2168-2177, Florence, Italy.

Polasik, M. et al, (2010). Time Efficiency Of Point-Of-Sale Payment Methods: Preliminary Results. Journal of Internet Banking and Commerce. vol. 15, no.3

Wang H., Cao J., Zhang Y. (2020) Using RBAC to Secure Payment Process in Cloud. In: Access Control Management in Cloud Environments. Springer, Cham.

Wilhelm, H., 1976. Datenübertragung (in German). Millitärverlag, Berlin, Germany

Wilhelm, H., 2018. Calculation of Error Structures in Binary Channels with Memory. Books on Demand, Norderstedt, 2018, Germany

Zhang, J., Shen, F., Waguespack, Y., 2016. Incorporating Generating Functions to Computational Science Education. In International Conference on Com- putational Science and Computational Intelligence (CSCI)., pages 315-320, Las Vegas USA

Zukerman, M., Neame, T. D., Addie, R. G., 2003. Internet traffic modeling and future technology implications. In IEEE INFOCOM 2003. Twenty-second Annual Joint Conference of the IEEE Computer and Communications Societies (IEEE Cat. No.03CH37428), volume 1, pages 587-596 